\title{
Hierarchical Modelling and Model Adaptivity for Gas Flow on Networks
}

\author{
Pia Bales, Oliver Kolb, and Jens Lang \\ Technische Universität Darmstadt \\ Schloßgartenstr. 7, 64289 Darmstadt, Germany
}

\begin{abstract}
We are interested in the simulation and optimization of gas transport in networks. Different regions of the network may be modelled by different equations. There are three models based on the Euler equations that describe the gas flow in pipelines qualitatively different: a nonlinear model, a semilinear model and a stationary also called algebraic model. For the whole network, adequate initial and boundary values as well as coupling conditions at the junctions are needed. Using adjoint techniques, one can specify model error estimators for the simplified models. A strategy to adaptively apply the different models in different regions of the network while maintaining the accuracy of the solution is presented.
\end{abstract}

Keywords: model adaptivity, adjoint equations, gas flow.

\section{Introduction}

During the last years, there has been intense research in the field of simulation and optimization of gas transport in networks [234489. The equations describing the transport of gas in pipelines are based on the Euler equations, a hyperbolic system of nonlinear partial differential equations, mainly consisting of the conservation of mass, momentum and energy. The transient flow of gas may be described appropriately by equations in one space dimension. For the whole network, adequate initial and boundary values as well as coupling conditions at the junctions are needed.

Although solving one-dimensional equations does not pose a challenge, the complexity increases with the size of the network. Thus, we present a hierarchy of models that describe the flow of gas in pipelines qualitatively different: The most detailed model we use consists of the isothermal Euler equations (continuity equation and momentum equation). A common simplification of the momentum equation leads to a semilinear model, which is only valid if the velocity of the gas is much less than the speed of sound, that is, $|v| \ll c$. Further simplifications lead to the steady state model. Obviously, simplified models are sufficient in network regions with low activity in the gas transport, while sophisticated models should be used to resolve high solution dynamics accurately. Since the whole network behaviour can change in both space and time, an automatic steering of the model hierarchy is essential. Existent software packages like SIMONE [11]

G. Allen et al. (Eds.): ICCS 2009, Part I, LNCS 5544, pp. 337-346, 2009.

(C) Springer-Verlag Berlin Heidelberg 2009 
may use stationary as well as transient models for the simulation. However, for the simulation process one model has to be chosen. The different models are introduced in Sect. 2. The modelling of the network as well as the boundary and coupling conditions are presented in Sect. 3.

In order to estimate the model error of the simplified models, that is, of the semilinear and the steady state model with respect to some quantity of interest, one has to solve adjoint systems on the network. For the adjoint equations, appropriate coupling conditions are required, which are introduced in Sect. 4. There, we also present a strategy, how to decide in which regions of the network which model has to be used to reduce the complexity of the whole problem, whereas the accuracy of the solution is maintained. We give numerical examples of this algorithm in Sect. 5.

\section{Model Hierarchy}

In this section, we introduce a hierarchy consisting of three different models. Each model results from the previous one by making further simplifying assumptions 1. The most complex model is the nonlinear model followed by the linear model. The most simple model used is the algebraic model (see Fig. 1).

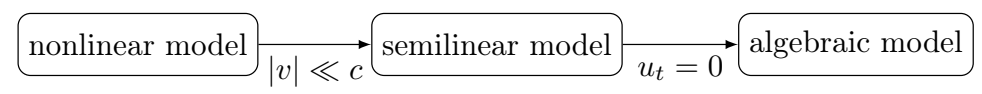

Fig. 1. Model hierarchy

\subsection{Nonlinear Model}

The isothermal Euler equations, which describe the flow of gas, consist of the continuity and the momentum equation:

$$
\frac{\partial \rho}{\partial t}+\frac{\partial(\rho v)}{\partial x}=0, \quad \frac{\partial(\rho v)}{\partial t}+\frac{\partial\left(\rho v^{2}\right)}{\partial x}+\frac{\partial p}{\partial x}=-g \rho h^{\prime}-\frac{\lambda}{2 d} \rho|v| v
$$

together with the equation of state for real gases $\rho=\frac{p}{z(p, T) R T}$.

Here, $\rho$ denotes the density, $v$ the velocity of the gas, $p$ the pressure, $g$ the gravity constant, $h^{\prime}$ the slope of the pipe, $\lambda$ the friction coefficient, $d$ the diameter of the pipe, $R$ the (special) gas constant, $T$ the temperature of the gas (assumed to be constant) and $z=z(p, T)$ the compressibility factor.

For the sake of simplicity, we assume the pipe to be horizontal and the compressibility factor $z$ to be constant. This results in a simplified equation of state $\rho=\frac{p}{c^{2}}$ with constant speed of sound $c=\sqrt{R T}$. Since the mass flow $M$ can be traced back to the flow rate under standard conditions $\left(M=\rho v A=\rho_{0} q\right)$, the system can be rewritten in the following way:

$$
p_{t}+\frac{\rho_{0} c^{2}}{A} q_{x}=0, \quad q_{t}+\frac{A}{\rho_{0}} p_{x}+\frac{\rho_{0} c^{2}}{A}\left(\frac{q^{2}}{p}\right)_{x}=-\frac{\lambda \rho_{0} c^{2}|q| q}{2 d A p} .
$$


Here, $\rho_{0}$ and $q$ denote density and flow rate under standard conditions (1 atm air pressure, temperature of $\left.0{ }^{\circ} \mathrm{C}\right), A$ the cross-sectional area of the pipe.

The characteristic speeds are the eigenvalues of the Jacobian of the system, which are $\lambda_{1 / 2}(u)=v \mp c$. Hence, for subsonic flow, the characteristics travel in opposite directions with characteristic speeds $\lambda_{1 / 2}$ depending on the velocity of the gas.

\subsection{Semilinear Model}

If the speed $v$ of the gas is much smaller than the speed of sound, we can neglect the nonlinear term in the spatial derivative of the momentum equation in (1). Together with the equation of state as above, this yields a semilinear model

$$
\begin{aligned}
& u_{t}+\mathbf{A} u_{x}=\psi(u) \\
& \text { with } u=\left(\begin{array}{l}
p \\
q
\end{array}\right), \mathbf{A}=\left(\begin{array}{cc}
0 & \frac{c^{2} \rho_{0}}{A} \\
\frac{A}{\rho_{0}} & 0
\end{array}\right) \text { and } \psi(u)=\left(\begin{array}{c}
0 \\
-\frac{\lambda \rho_{0} c^{2}|q| q}{2 d A p}
\end{array}\right) .
\end{aligned}
$$

For this model the characteristic speeds are $\lambda_{1 / 2}=\mp c$. Thus, information always travels in both directions with sonic speed.

\subsection{Algebraic Model}

A further simplification leads to the stationary model: Setting the time derivatives in (3) to zero results in

$$
q_{x}=0, \quad \frac{A}{\rho_{0}} p_{x}=-\frac{\lambda \rho_{0} c^{2}|q| q}{2 d A p} .
$$

Thus, $q$ is constant in space and the exact solution for $p$ is

$$
p(x)=\sqrt{p\left(x_{0}\right)^{2}+\frac{\lambda \rho_{0}^{2} c^{2}|q| q}{d A^{2}}\left(x_{0}-x\right)} .
$$

Here, $p\left(x_{0}\right)$ denotes the pressure at an arbitrary point $x_{0} \in[0, L]$. Setting $x_{0}=0$, that is, $p\left(x_{0}\right)=p(0)=p_{\text {in }}$ at the inbound of the pipe, and $x=L$, that is, $p(x)=p(L)=p_{\text {out }}$ at the end of the pipe, yields the algebraic model [10].

For the other two models, we computed characteristic speeds at which information propagates in different directions. Since this model is stationary, information given at any place instantaneously influences all other points.

\section{Modelling of the Network}

We now want to describe the gas flow on networked pipelines. For this purpose, we model the network as a directed graph $\mathcal{G}=(\mathcal{J}, \mathcal{V})$ with edges $\mathcal{J}$ (pipes) and vertices $\mathcal{V}$ (nodes, branching points). Each edge $j \in \mathcal{J}$ is defined as an interval $\left(x_{j}^{a}, x_{j}^{b}\right)$ with a direction from $x_{j}^{a}$ to $x_{j}^{b}$. Of course, all intervals are disjoint. Then, for any inner node $v$, we can define two sets of edges. Let the set of 


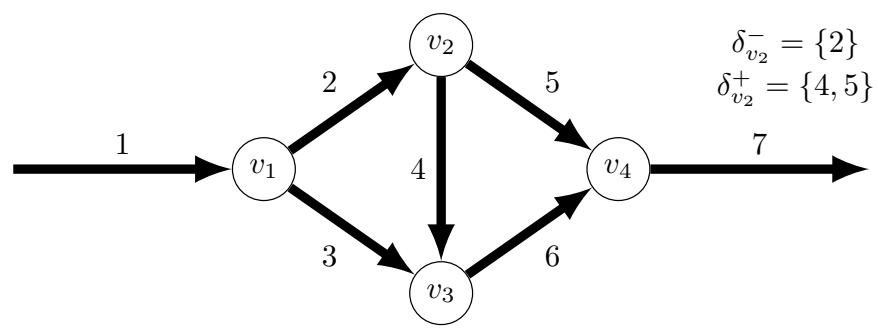

Fig. 2. A small network; the ingoing pipes of node $v_{2}$ are $\delta_{v_{2}}^{-}=\{2\}$ and the set of outgoing pipes is $\delta_{v_{2}}^{+}=\{4,5\}$

ingoing pipes be denoted by $\delta_{v}^{-}$, that is, the set of any edge $j \in \mathcal{J}$ with endpoint $x_{j}^{b}$ being adjacent to $v$. Then, analogously, $\delta_{v}^{+}$denotes the set of outgoing pipes (see Fig. 2). Inside each pipe, one of the models described above holds. In order to obtain a unique solution, we have to pose coupling conditions at the inner nodes of the network as well as boundary conditions at the sources and sinks.

\subsection{Coupling Conditions}

A first coupling condition is the conservation of mass at each inner node. Let $v \in \mathcal{V}$ be a node with ingoing pipes $j \in \delta_{v}^{-}$and outgoing pipes $i \in \delta_{v}^{+}$. Then, Kirchhoff's law (conservation of mass) yields

$$
\sum_{j \in \delta_{v}^{-}} q\left(x_{j}^{b}, t\right)=\sum_{i \in \delta_{v}^{+}} q\left(x_{i}^{a}, t\right) .
$$

Next, we need further coupling conditions and there are several possibilities. The most common condition used is the equality of pressure at the node $v$ as pointed out in [3]: $p\left(x_{i}^{b}, t\right)=p\left(x_{j}^{a}, t\right) \forall i \in \delta_{v}^{+}, j \in \delta_{v}^{-}$.

\subsection{Boundary Conditions}

Let $\mathcal{J}_{\text {in }}$ denote the set of ingoing pipes of the network, i.e. the pipes connecting the sources with the network and let $\mathcal{J}_{\text {out }}$ denote the set of outgoing pipelines connected with sinks.

Since for subsonic flow the characteristics of the nonlinear model propagate in different directions and for the semilinear model the characteristics always propagate in reverse directions, one can prescribe the characteristic variables only on opposing sides of a pipe. Thus, there are some limitations on the boundary conditions of the edges.

One possibility is to specify the pressure $p$ at one end of the pipe and the flow rate $q$ at the other. So, we usually prescribe the pressure at $x_{j}^{a}, j \in \mathcal{J}_{\text {in }}$ (sources) and the flow rate at $x_{j}^{b}, j \in \mathcal{J}_{\text {out }}$ (sinks). 


\subsection{Gas Flow on the Network}

We can now describe the flow of gas on the network. With the notations $\Omega=$ $\bigcup_{j \in \mathcal{J}}\left[x_{j}^{a}, x_{j}^{b}\right]$ and $Q:=\Omega \times(0, T)$, the equations for the nonlinear model read as follows:

$$
\begin{array}{ll}
u_{t}+f(u)_{x}=\psi(u) & \text { in } Q \\
p(x, 0)=p_{0}(x) & \text { in } \Omega \\
q(x, 0)=q_{0}(x) & \text { in } \Omega \\
p\left(x_{i}^{a}, t\right)=w_{i}(t) & i \in \mathcal{J}_{\text {in }}, t \in(0, T) \\
q\left(x_{i}^{b}, t\right)=v_{i}(t) & i \in \mathcal{J}_{\text {out }}, t \in(0, T) \\
p\left(x_{i}^{b}, t\right)=p\left(x_{j}^{a}, t\right) & \forall v \in \mathcal{V}, i \in \delta_{v}^{-}, j \in \delta_{v}^{+}, t \in(0, T) \\
\sum_{i \in \delta_{v}^{-}} q\left(x_{i}^{b}, t\right)=\sum_{i \in \delta_{v}^{+}} q\left(x_{i}^{a}, t\right) & \forall v \in \mathcal{V}, t \in(0, T) \\
w_{i}(t)>0 & i \in \mathcal{J}_{\text {in }}, t \in(0, T) .
\end{array}
$$

For the semilinear and the algebraic model, the equations are analogous to (5) with the corresponding PDE or algebraic equation in the first line. The boundary conditions $p\left(x_{i}^{a}, t\right), i \in \mathcal{J}_{\text {in }}, t \in(0, T)$ and $q\left(x_{i}^{b}, t\right), i \in \mathcal{J}_{\text {out }}, t \in(0, T)$ are determined by control variables/functions $w_{i}(t)$ and $v_{i}(t)$. Since the flow rate at the sinks is given by the consumers, the variable that can be controlled by us will only be the pressure at the sources.

\section{Adjoint Equations on the Network}

A possibility to achieve a compromise between the accuracy of the model and the computational costs is to use the more complex model only when necessary. Using the solution of adjoint equations as done in [5]6], we deduce a model error estimator to measure the influence of the model on a user-defined output functional.

Let the functional $M$ be of the form

$$
M(u)=\int_{Q} N(u) \mathrm{d} t \mathrm{~d} x+\sum_{i \in \mathcal{J}_{\text {in }}} \int_{0}^{T} N_{x_{i}^{a}}(q) \mathrm{d} t+\sum_{i \in \mathcal{J}_{\text {out }}} \int_{0}^{T} N_{x_{i}^{b}}(p) \mathrm{d} t+\int_{\Omega} N_{T}(u) \mathrm{d} x .
$$

As pointed out in [6], we only need to solve the dual problem of the simplified models in order to obtain a first order error estimator. Let $\xi=\left(\xi_{1}, \xi_{2}\right)^{T}$ be the solution of the dual problem of the semilinear model (3) or the algebraic model (44) with respect to the functional $M$.

For a given solution $u^{*}=\left(p^{*}, q^{*}\right)^{T}$ of the semilinear equations, the adjoint system on the network reads as follows:

$$
\begin{array}{ll}
\xi_{t}+\mathbf{A}^{T} \xi_{x}=-\partial_{u} \psi\left(u^{*}\right)^{T} \xi-\partial_{u} N\left(u^{*}\right)^{T} & \text { in } Q \\
\xi(\cdot, T)=\partial_{u} N_{T}\left(u^{*}(\cdot, T)\right)^{T} & \text { in } \Omega \\
\xi_{1}\left(x_{i}^{a}, t\right)=-\frac{A_{i}}{\rho_{0} c^{2}} \partial_{q} N_{x_{i}^{a}}\left(q^{*}\left(x_{i}^{a}, t\right)\right) & i \in \mathcal{J}_{\text {in }}, t \in(0, T) \\
\xi_{2}\left(x_{i}^{b}, t\right)=\frac{\rho_{0}}{A_{i}} \partial_{p} N_{x_{i}^{b}}\left(p^{*}\left(x_{i}^{b}, t\right)\right) & i \in \mathcal{J}_{\text {out }}, t \in(0, T) .
\end{array}
$$


The adjoint system for the algebraic equations is similar to that of the semilinear model, only that the time derivative and the initial conditions vanish. Thus, one cannot measure the influence of the algebraic model at the final time $T$, which means that the last term of 6 , i.e. $\int_{\Omega} N_{T}(u) \mathrm{d} x$, has to be left out. For the adjoint systems, one also has to specify coupling conditions. Conservation of mass and equality of pressure at the node $v$ yield for the adjoint variables:

$$
\begin{aligned}
\frac{1}{A_{i}} \xi_{1}\left(x_{i}^{b}, t\right) & =\frac{1}{A_{j}} \xi_{1}\left(x_{j}^{a}, t\right), & & i \in \delta_{v}^{-}, j \in \delta_{v}^{+}, t \in(0, T), \\
\sum_{i \in \delta_{v}^{-}} A_{i} \xi_{2}\left(x_{i}^{b}, t\right) & =\sum_{j \in \delta_{v}^{+}} A_{j} \xi_{2}\left(x_{j}^{a}, t\right), & & t \in(0, T) .
\end{aligned}
$$

\subsection{Error Estimators}

We now use the adjoint equations to assess the simplified models with respect to the quantity of interest. Let $u=(p, q)^{T}$ be the solution of the nonlinear model (2) and $u^{h}=\left(p^{h}, q^{h}\right)^{T}$ the discretized solution of the semilinear model (3). Then the difference between the output functional of $u, M(u)$, and $M\left(u^{h}\right)$ is

$$
\begin{aligned}
M(u)-M\left(u^{h}\right)= & \int_{Q} N(u)-N\left(u^{h}\right) \mathrm{d} t \mathrm{~d} x+\sum_{i \in \mathcal{J}_{\text {in }}} \int_{0}^{T} N_{x_{i}^{a}}(q)-N_{x_{i}^{a}}\left(q^{h}\right) \mathrm{d} t \\
& +\sum_{i \in \mathcal{J}_{\text {out }}} \int_{0}^{T} N_{x_{i}^{b}}(p)-N_{x_{i}^{b}}\left(p^{h}\right) \mathrm{d} t+\int_{\Omega} N_{T}(u)-N_{T}\left(u^{h}\right) \mathrm{d} x .
\end{aligned}
$$

Taylor expansion of first order yields

$$
\begin{aligned}
= & \int_{Q} \partial_{u} N\left(u^{h}\right)\left(u-u^{h}\right) \mathrm{d} t \mathrm{~d} x+\sum_{i \in \mathcal{J}_{\text {in }}} \int_{0}^{T} \partial_{q} N_{x_{i}^{a}}\left(q^{h}\right)\left(q-q^{h}\right) \mathrm{d} t \\
& +\sum_{i \in \mathcal{J}_{\text {out }}} \int_{0}^{T} \partial_{p} N_{x_{i}^{b}}\left(p^{h}\right)\left(p-p^{h}\right) \mathrm{d} t+\int_{\Omega} \partial_{u} N_{T}\left(u^{h}\right)\left(u-u^{h}\right) \mathrm{d} x+\text { H.O.T. }
\end{aligned}
$$

with H.O.T . being higher order terms. Inserting the solution $\xi$ of the adjoint system (7), we get a first order error estimator for the model and the discretization error respectively as in [6]:

$$
M(u)-M\left(u^{h}\right) \approx \eta_{m}+\eta_{h}
$$

with the estimators $\eta_{m}$ and $\eta_{h}$ as follows:

$$
\begin{aligned}
\eta_{m}^{n l-s l} & =\int_{Q}-\xi^{T}\left(\begin{array}{c}
0 \\
\frac{\rho_{0} c^{2}\left(q^{h}\right)^{2}}{A p^{h}}
\end{array}\right)_{x} \mathrm{~d} x \mathrm{~d} t \\
\eta_{h}^{n l-s l} & =\int_{Q} \xi^{T}\left(-u_{t}^{h}-\mathbf{A} u_{x}^{h}+\psi\left(u^{h}\right)\right) \mathrm{d} x \mathrm{~d} t .
\end{aligned}
$$


Since the algebraic model can be solved exactly, the discretization error disappears and one only gets an estimator for the model error

$$
\eta_{m}^{s l-a l g}=\int_{Q}-\xi^{T}\left(\begin{array}{l}
p \\
q
\end{array}\right)_{t} \mathrm{~d} x \mathrm{~d} t
$$

with $\xi$ being the solution of the adjoint equations either of the semilinear model (7) or of the algebraic model. Here, $u=\left(\begin{array}{c}p \\ q\end{array}\right)$ denotes the solution of the stationary model (4).

\subsection{Adaptive Switching Strategy}

With the estimators defined above we may now derive a strategy to switch adaptively between the models. For this, we divide the time interval $(0, T)$ into equal subintervals $\left(T_{k-1}, T_{k}\right), k=1, \ldots, N_{B}$, with $T_{0}=0$ and $T_{N_{B}}=T$. Thus, we can split up the computational domain $Q=\Omega \times(0, T)$ into $N_{B}$ blocks $Q_{k}=\Omega \times\left(T_{k-1}, T_{k}\right), k=1, \ldots, N_{B}$, of equal size (see Fig. $3(\mathrm{a})$.

We start with simulating the first block $Q_{1}$. Each pipe is assigned to one of the three models. Then, we solve the corresponding adjoint system in order to estimate the model error using (9) and (11) respectively. The model error estimator on $Q_{1}$ can now be computed for each pipe separately. For the semilinear case (9) this reads as follows.

$$
\begin{aligned}
& \eta_{m}=\sum_{k=1}^{N_{B}} \int_{Q_{k}}-\xi^{T}\left(\begin{array}{c}
0 \\
\frac{\rho_{0} c^{2}\left(q^{h}\right)^{2}}{A p^{h}}
\end{array}\right)_{x} \mathrm{~d} x \mathrm{~d} t \\
& =\sum_{k=1}^{N_{B}} \sum_{j \in \mathcal{J}} \int_{T_{k-1}}^{T_{k}} \int_{x_{j}^{a}}^{x_{j}^{b}}-\xi^{T}\left(\begin{array}{c}
0 \\
\frac{\rho_{0} c^{2}\left(q^{h}\right)^{2}}{A p^{h}}
\end{array}\right)_{x} \mathrm{~d} x \mathrm{~d} t=: \sum_{k=1}^{N_{B}} \sum_{j \in \mathcal{J}} \eta_{m}(k, j)
\end{aligned}
$$

with the "local" estimators $\eta_{m}(k, j)$.

Given a tolerance TOL, one can decide in which pipe the model used is appropriate and in which it is not. We want to accept the model if the relative deviation of the simpler model $u^{h}$ from the exact solution of the more complex model $u$ is below TOL, that is, $\left|M(u)-M\left(u^{h}\right)\right| /\left|M\left(u^{h}\right)\right| \leq$ TOL. Provided that the discretization error is nonsignificant compared to the model error, we can approximate $\left|M(u)-M\left(u^{h}\right)\right|$ by $\left|\eta_{m}\right|$, which yields

$$
\left|\eta_{m}\right| \leq \text { TOL }\left|M\left(u^{h}\right)\right|
$$

Just like the error estimator $\eta_{m}$, we can evaluate the target functional $M$ at every pipe $j \in \mathcal{J}$ and every time interval $\left(T_{k-1}, T_{k}\right), k=1, \ldots, N_{B}$ individually, giving $M_{k, j}$, that is, $M\left(u^{h}\right)=\sum_{k=1}^{N_{B}} \sum_{j \in \mathcal{J}} M_{k, j}\left(u^{h}\right)$. Thus, for inequality (12) to hold, it suffices to claim

$$
\left|\eta_{m}(k, j)\right| \leq \text { TOL }\left|M_{k, j}\left(u^{h}\right)\right|, \quad \forall k \in\left\{1, \ldots, N_{B}\right\}, j \in \mathcal{J} .
$$




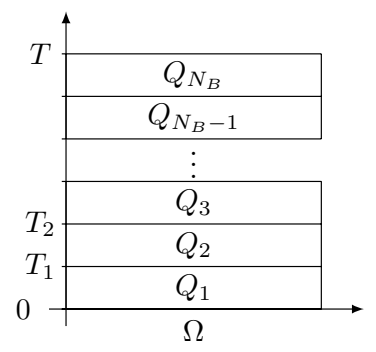

(a)

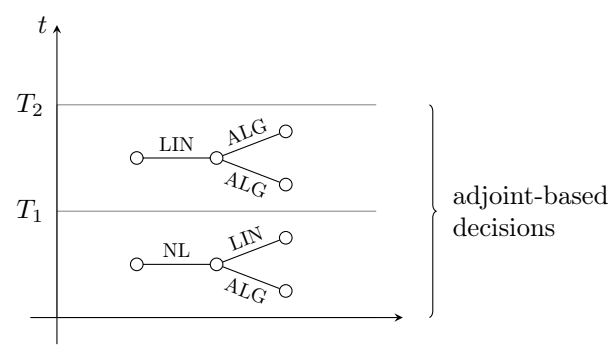

(b)

Fig. 3. (a) Partition of the computational domain; (b) Scheme of the adaptive switching $(\mathrm{ALG}=$ algebraic model, $\mathrm{LIN}=$ semilinear model, $\mathrm{NL}=$ nonlinear model $)$

If any of the estimators $\eta_{m}(k, j)$ violates (13), the computation of this time interval has to be repeated and the models used in these pipes have to be exchanged by a more complex model. For those pipes of which the estimators fulfil inequality (13), one can evaluate the estimators "downwards". If these also fulfil (13), a more simple model may be used in the next time step. For a scheme of the switching strategy, see Fig. 3(b).

\section{$5 \quad$ Numerical Results}

We give an example of the algorithm for a small network. It consists of nine pipes Le1 to Le9, one source Qu, four inner nodes M1 to M4 and three sinks Se1 to Se3 (see Fig. 4(a)).

All pipes have a diameter of $1 \mathrm{~m}$ and a roughness of $5 \cdot 10^{-5} \mathrm{~m}$. The lengths of all except two pipes is $10 \mathrm{~km}$. The pipes Le4 and Le6 are both $5 \mathrm{~km}$ long. The simulation time totals $T=14400 \mathrm{~s}$ with time step size $\Delta t=5 \mathrm{~s}$. The block size was chosen the size of a time step.

As boundary conditions we use constant pressure at the source $\mathrm{Qu}$ and a constant flow rate at sinks Se2 and Se3. The gas consumption at sink Se1 is chosen time-dependently with initially $q\left(x_{4}^{b}, t\right)=250 \frac{\mathrm{m}^{3}}{\mathrm{~s}}$ for $t \leq 100 \mathrm{~s}$ and $q\left(x_{4}^{b}, t\right)=300 \frac{\mathrm{m}^{3}}{\mathrm{~s}}$ for $t \geq 105 \mathrm{~s}$ and a linear increase in-between. The initial conditions are chosen stationary (Fig. 4(b)). The target functional used is $M(p, q)=\int_{Q} p \mathrm{~d} x \mathrm{~d} t$, the "Quantity of Interest" is thus the pressure measured over the whole network.

In this setting, only two models were used: the semilinear model (3) and the algebraic model (44). A reference solution was computed using the semilinear model. The equations were solved using an implicit box scheme [7]. Figure 5] shows the simulation process at times $115 \mathrm{~s}$ and $7075 \mathrm{~s}$.

Figure 6 compares the pressure of the adaptive solution with the reference solution at sink Se1 for two different tolerances. 


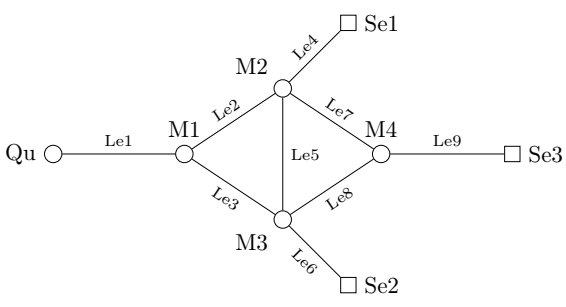

(a)

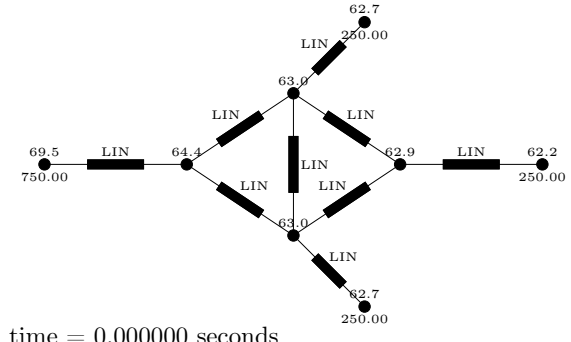

(b)

Fig. 4. (a) A small network; (b) Initial conditions; at every node the pressure $p$ is given, at the sources and sinks additionally the flow rate $q$ is specified below

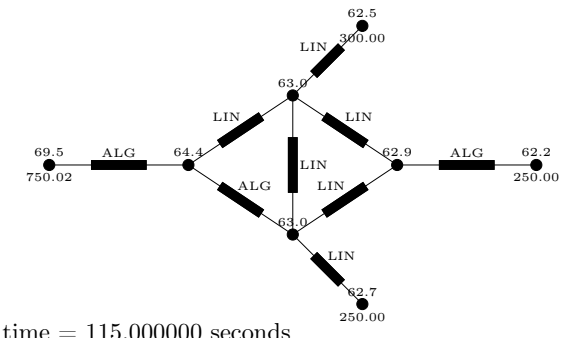

(a)

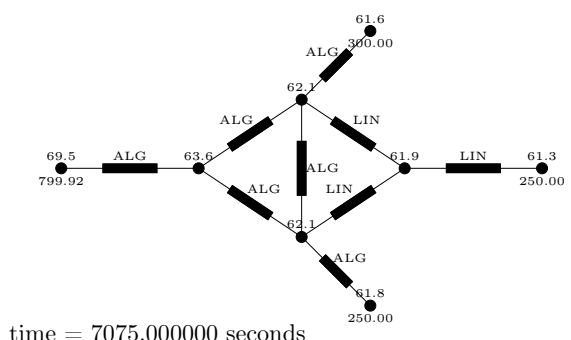

(b)

Fig. 5. Two snapshots of the simulation process using the adaptive switching strategy
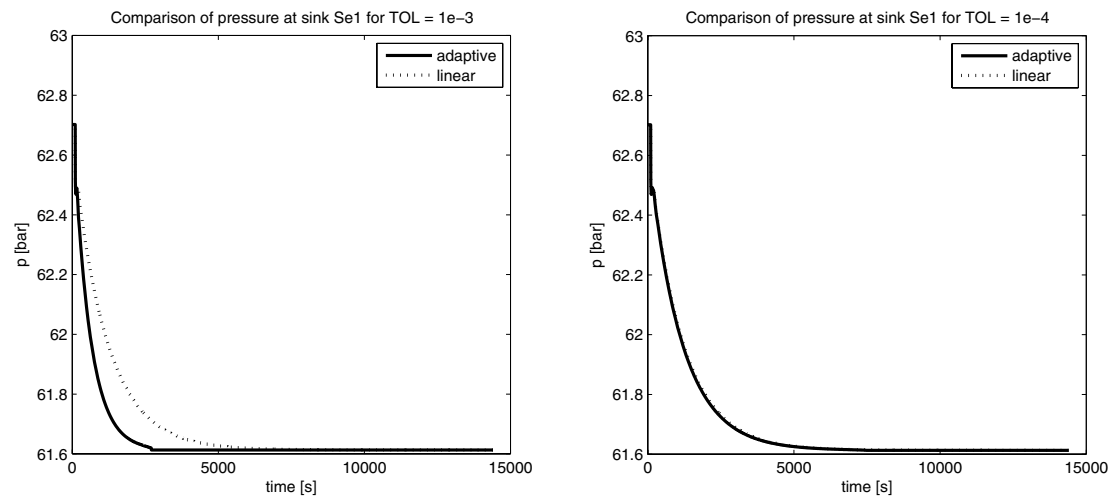

Fig. 6. Comparison of the pressure with the reference solution at sink Se1 for different values of TOL

\section{Summary}

We introduced a model hierarchy for the simulation of gas transport in networked pipelines. This hierarchy consists of a nonlinear and a semilinear system of 
hyperbolic partial differential equations and of an algebraic steady state model. We discussed coupling and boundary conditions for the wellposedness of the whole system. For the network, adjoint equations as well as adjoint coupling conditions were given that allow us to valuate the different models with respect to a quantity of interest. An algorithm was developed that switches adaptively between the three models using model error estimators deduced from the adjoint systems. The additional computational effort is approximately that of solving the original system. In the case of locally restricted dynamical effects we observed for a test network a significant reduction of complexity, while a certain accuracy is maintained. As a side result we gain an estimator for the discretization error for free.

Based on our results we want to proceed in testing the switching strategy for more complex systems including compressor stations and valves. Furthermore, an integration into an optimization framework is planned.

Acknowledgments. This paper was supported by the German Research Foundation (DFG) under the grant LA1372/5-1.

\section{References}

1. Bales, P.: Hierarchische Modellierung der Eulerschen Flussgleichungen in der Gasdynamik. Diploma thesis, TU Darmstadt (2005)

2. Bales, P., Geißler, B., Kolb, O., Lang, J., Martin, A., Morsi, A.: Comparison of Linear and Nonlinear Optimization of Transient Gas Networks. Preprint No. 2552, TU Darmstadt (2008)

3. Banda, M., Herty, M., Klar, A.: Coupling conditions for gas networks governed by the isothermal euler equations. NHM 1(2), 295-314 (2006)

4. Banda, M., Herty, M., Klar, A.: Gas flow in pipeline networks. NHM 1(1), 41-56 (2006)

5. Becker, R., Rannacher, R.: An optimal control approach to a posteriori error estimation in finite element methods. Acta numerica 10, 1-102 (2001)

6. Braack, M., Ern, A.: A posteriori control of modeling errors and discretization errors. SIAM Multiscale Model. Simul. 1(2), 221-238 (2003)

7. Kolb, O., Lang, J., Bales, P.: Adaptive linearization for the optimal control problem of gas flow in pipeline networks. Preprint No. 2553, TU Darmstadt (2008)

8. Martin, A., Möller, M., Moritz, S.: Mixed integer models for the stationary case of gas network optimization. Math. Prog. 105, 563-582 (2006)

9. Moritz, S.: A Mixed Integer Approach for the Transient Case of Gas Network Optimization. PhD thesis, TU Darmstadt (2006)

10. Sekirnjak, E.: Transiente Technische Optimierung. Concept, PSI AG (2000)

11. SIMONE, http://www.simone.eu/simone-simonesoftware.asp 